\title{
QUASI-MULTIPLIERS OF HILBERT AND BANACH $C^{*}$-BIMODULES
}

\author{
ALEXANDER PAVLOV, ULRICH PENNIG and THOMAS SCHICK
}

\begin{abstract}
Quasi-multipliers for a Hilbert $C^{*}$-bimodule $V$ were introduced by L. G. Brown, J. A. Mingo, and N.-T. Shen [3] as a certain subset of the Banach bidual module $V^{* *}$. We give another (equivalent) definition of quasi-multipliers for Hilbert $C^{*}$-bimodules using the centralizer approach and then show that quasi-multipliers are, in fact, universal (maximal) objects of a certain category. We also introduce quasi-multipliers for bimodules in Kasparov's sense and even for Banach bimodules over $C^{*}$-algebras, provided these $C^{*}$-algebras act non-degenerately. A topological picture of quasimultipliers via the quasi-strict topology is given. Finally, we describe quasi-multipliers in two main situations: for the standard Hilbert bimodule $l_{2}(A)$ and for bimodules of sections of Hilbert $C^{*}$-bimodule bundles over locally compact spaces.
\end{abstract}

\section{Introduction}

There are several equivalent ways to introduce quasi-multipliers (left as well as right and (double) multipliers) for a $C^{*}$-algebra $A$. It may be done in terms of centralizers ([4]), via universal representations treating $A$ as a $C^{*}$-subalgebra of its enveloping von Neumann algebra $A^{* *}$ (cf., e.g., [15, § 3.12]) and by a categorical approach describing multipliers as universal objects in suitable categories ([11, Ch. 2], [13]). These theories were extended to the category of Hilbert $C^{*}$-(bi)modules. More precisely, in this context multipliers were defined and studied in [2], [16], left multipliers in [8] and quasi-multipliers in [3]. These concepts coincide with the theories for $C^{*}$-algebras in the particular situation when the Hilbert (bi)module under consideration is nothing else but the underlying $C^{*}$-algebra.

Our aim in this work is to define and study quasi-multipliers for Hilbert $C^{*}$-bimodules, Hilbert bimodules in Kasparov's sense and, more generally, even for Banach bimodules over $C^{*}$-algebras, on which both algebras act nondegenerately. For Hilbert $C^{*}$-bimodules our definition of quasi-multipliers differs from the one of [3], but, as we show, these definitions are actually equivalent. We introduce quasi-multipliers using the centralizer approach, and then show that these objects are, in fact, universal (maximal) objects of some categories. Note that in [3] quasi-multipliers of a Hilbert $C^{*}$-bimodule $V$ are

Received 7 April 2010. 
considered as a certain subset of the Banach bidual module $V^{* *}$ that allows to characterize embeddings of Hilbert $C^{*}$-bimodules into $C^{*}$-algebras, [3, Theorem 4.3]. We study also the quasi-strict topology and give the topological picture of quasi-multipliers in terms of this topology.

Finally, we give the description for quasi-multipliers in two main situations: for standard bimodules $l_{2}(A)$ (actually, we obtain a much more general result concerning quasi-multipliers of infinite direct sums of bimodules) and for the "commutative" case. The latter means that, for a given locally compact space $X$ and a Hilbert $A-B$ bimodule $V$, we treat quasi-multipliers of the Hilbert $A_{0}(X)$ $B_{0}(X)$-bimodule $\mathscr{V}_{0}(X)=C_{0}(X, \mathscr{V})$. These are the continuous sections of a Hilbert $A$ - $B$-bimodule bundle $\mathscr{V}$ over $X$ with typical fiber $V$. Moreover, $A_{0}(X)$ and $B_{0}(X)$ denote the set of continuous $A$-valued and $B$-valued functions on $X$ vanishing at infinity.

\section{Quasi-multipliers of Hilbert $C^{*}$-bimodules}

Given a $C^{*}$-algebra $A$ and a Banach space $Q$, recall that $Q$ is said to be an involutive Banach space if it is equipped with a sesqui-linear involution $*: Q \rightarrow Q$ such that $\left\|q^{*}\right\|=\|q\|$ for any $q \in Q$. We will also need some definitions of [13].

Definition 2.1. An involutive Banach space $Q$ with involution $q \mapsto q^{*}$ is called an A-bimodule if there is a map, which is conjugate linear in the first variable and linear in the second variable

$$
A \times Q \rightarrow Q, \quad(a, q) \mapsto a \triangleleft q,
$$

and a bilinear map

$$
Q \times A \rightarrow Q, \quad(q, a) \mapsto q \triangleright a
$$

such that

$$
\begin{aligned}
& (b a) \triangleleft q=a \triangleleft(b \triangleleft q), \quad(a \triangleleft q \triangleright b)^{*}=b \triangleleft q^{*} \triangleright a, \\
& q \triangleright(a b)=(q \triangleright a) \triangleright b, \quad \quad\|a \triangleleft q\| \leq\|a\|\|q\|, \\
& (a \triangleleft q) \triangleright b=a \triangleleft(q \triangleright b), \quad \quad\|q \triangleright b\| \leq\|q\|\|b\|
\end{aligned}
$$

for all $a, b \in A, q \in Q$.

Definition 2.2. Let $Q$ be a bimodule over $A$. Moreover assume that $A \subset$ $Q$ is an involutive Banach subspace. $A$ is said to be a quasi-ideal of $Q$ if

$$
a \triangleleft b=a^{*} b, \quad b \triangleright a=b a \quad \text { for } \quad a, b \in A
$$

and $A \triangleleft q \triangleright A \subset A$ for any $q \in Q$. 
Proposition 2.3 ([13, comments to Definition 3]). Let $A \subset Q$ be a quasiideal and $Q^{(0)}=\{q \in Q: A \triangleleft q \triangleright A=0\}$. Then $Q^{(0)}$ is a sub-bimodule of $Q$ and the following conditions are equivalent.

(i) $Q^{(0)}=0$;

(ii) For any A-sub-bimodule $X$ of $Q$ the condition $X \cap A=\{0\}$ implies $X=\{0\}$.

Definition 2.4. A quasi-ideal $A \subset Q$ is essential if it satisfies one of the equivalent conditions above.

Definition 2.5. A quasi-ideal $A \subset Q$ is strictly essential if

$$
\sup \{\|a \triangleleft q \triangleright b\|: a, b \in A,\|a\| \leq 1,\|b\| \leq 1\}=\|q\|
$$

for all $q \in Q$.

Quasi-multipliers $Q M(A)$ of $A$ may be, actually, introduced in several equivalent ways, but we prefer here to use their original definition in terms of quasi-centralizers (cf. [4]).

Definition 2.6. A quasi-multiplier of $A$ is a bilinear bounded map $q: A \times$ $A \rightarrow A$ such that

$$
q(c a, b d)=c q(a, b) d \quad \text { for } \quad a, b, c, d \in A .
$$

The set of quasi-multipliers $Q M(A)$ is an involutive Banach space with respect to the operator norm $\|q\|:=\sup \{\|q(a, b)\|:\|a\| \leq 1,\|b\| \leq 1\}$ and the involution: $q^{*}(a, b)=q\left(b^{*}, a^{*}\right)^{*}$, where $a, b \in A, q \in Q M(A)$ (cf. [15, 3.12.2]).

Proposition 2.7 ([13]). A is embedded into $Q M(A)$ as an involutive Banach subspace via the $*$-inclusion

$$
a \mapsto q_{a}, \quad q_{a}(b, c)=b a c,
$$

$a, b, c \in A$. Moreover, $A$ is actually a strictly essential quasi-ideal of $Q M(A)$ and $Q M(A)$ is maximal (with respect to injective homomorphisms of involutive Banach spaces acting identically on A) among all quasi strictly essential extensions of $A$.

Now we are going to adopt the considerations of [2], [8] about double and left multipliers of Hilbert $C^{*}$-modules to introduce quasi-multipliers in the $C^{*}$-module context. But, as we saw before, even for $C^{*}$-algebras we need a bimodule structure for the definition of quasi-multipliers. Consequently, we 
need Hilbert $C^{*}$-bimodules (moreover, equipped with some involution) instead of usual Hilbert $C^{*}$-modules for the following considerations. Thus, we come to the following definition.

Definition 2.8. A Hilbert $A$-B-bimodule $V$ is both: a left Hilbert $A$ module and a right Hilbert $B$-module with commuting actions such that its left ${ }_{A}\langle\cdot, \cdot\rangle$ and right $\langle\cdot, \cdot\rangle_{B}$ inner products satisfy the condition

$$
{ }_{A}\langle x, y\rangle z=x\langle y, z\rangle_{B}
$$

for all $x, y, z \in V$. If $V$ is a Hilbert $A$-A-bimodule and a Banach involutive space such that

$$
(a x)^{*}=x^{*} a^{*}, \quad(x a)^{*}=a^{*} x^{*} \quad \text { for } \quad a \in A, x \in V,
$$

is said to be an involutive Hilbert A-bimodule.

The two norms defined on $V$, one from each inner product necessarily coincide by [3, Corollary 1.11].

EXAMPLE 2.9. Any $C^{*}$-algebra may be considered as an involutive Hilbert bimodule over itself with respect to the inner products ${ }_{A}\langle a, b\rangle=a b^{*}$ and $\langle a, b\rangle_{A}=a^{*} b$, where $a, b \in A$. Obviously, any free module $A^{n}$ is an involutive Hilbert bimodule. Observe, however, that the standard module $l^{2}(A)$ in general is not involutive, as was pointed out to us by the referee.

Example 2.10. Any right Hilbert $A$-module $V$ may be considered as a Hilbert $K(V)$-A-bimodule with respect to the inner product

$$
K(V)\langle x, y\rangle=x\langle y, \cdot\rangle_{A} .
$$

Example 2.11. Let $A$ be a $C^{*}$-subalgebra of a $C^{*}$-algebra $B$ and $E: B \rightarrow A$ be a conditional expectation, i.e., a surjective projection of norm one satisfying the following conditions:

$$
E(a b)=a E(b), \quad E(b a)=E(b) a, \quad E(a)=a,
$$

for $a \in A, b \in B$ (cf. [18]). Then $B$ (with its $C^{*}$-algebra involution) is an involutive pre-Hilbert $A$-bimodule with respect to the inner products ${ }_{A}\langle x, y\rangle=$ $E\left(x y^{*}\right)$ and $\langle x, y\rangle_{A}=E\left(x^{*} y\right)$. This module is Hilbert if and only if $E$ is topologically of index-finite type, i.e., the mapping $\left(K \cdot E-\mathrm{id}_{B}\right)$ is positive for some real number $K \geq 1$ (cf. [6], [7]). 
Definition 2.12. Given two $C^{*}$-algebras $A$ and $B$ and a Hilbert $A-B$ bimodule $V$, the quasi-multipliers of $V$ are defined as the set of all bounded $A$ - $B$-bilinear homomorphisms from $A \times B$ to $V$,

$$
Q M(V)=\operatorname{Hom}_{A, B}(A \times B, V),
$$

with norm $\|q\|:=\sup \{\|q(a, b)\| \mid a \in A, b \in B$ with $\|a\| \leq 1,\|b\| \leq 1\}$.

$V$ is isometrically embedded into $Q M(V)$ by the map

$$
\Gamma: V \rightarrow Q M(V), \quad \Gamma(x)(a, b)=a x b,
$$

and we will identify $V$ with its image under this embedding. If $V$ is an involutive Hilbert $A$ - $A$-bimodule, then $Q M(V)$ carries an involution $T^{*}(a, b)=$ $T\left(b^{*}, a^{*}\right)^{*}$ with respect to which quasi-multipliers $Q M(V)$ form an involutive Banach space.

REMARK 2.13. In [3] quasi-multipliers were defined via the bidual $V^{* *}$ of $V$ as a Banach space by the formula

$$
\widetilde{Q M}(V)=\left\{t \in V^{* *} \mid a t b \in V \text { for all } a \in A, b \in B\right\} .
$$

This definition actually coincides with the one above in the following sense. Clearly, every element $t \in \widetilde{Q M}(V)$ defines a bimodule homomorphism

$$
q_{t}: A \times B \longrightarrow V, \quad(a, b) \mapsto a t b .
$$

That means there is a linear map

$$
\varphi: \widetilde{Q M}(V) \rightarrow Q M(V), \quad t \mapsto q_{t},
$$

which, in fact, is an isometry, because

$$
\left\|q_{t}\right\|=\sup \{\|a t b\|:\|a\| \leq 1,\|b\| \leq 1\}=\|t\|
$$

for any $t \in \widetilde{Q M}(V)$ by [3, Lemma 4.1(iii)]. To see that $\varphi$ is surjective, let $q \in$ $Q M(V)$ be given, choose approximate units $\left\{e_{\alpha}\right\}$ in $A$ and $\left\{u_{\beta}\right\}$ in $B$. Then by [3, Lemma 4.1(iv)] there is $t \in \widetilde{Q M}(V)$ such that $q(a, b)=\lim _{\alpha, \beta} a q\left(e_{\alpha}, u_{\beta}\right) b=$ $a t b$. Such $t$ is just a $\sigma\left(V^{* *}, V^{*}\right)$ cluster point of the bounded net $\left\{q\left(e_{\alpha}, u_{\beta}\right)\right\}$, which has to exist by the Banach-Alaoglu theorem.

Definition 2.14. Given two Banach algebras $\mathscr{A}$ and $\mathscr{B}$, a Banach space $W$ is called a Banach- $\mathscr{A}-\mathscr{B}$-bimodule if it is equipped with a norm continuous left action of $\mathscr{A}$ and a norm continuous right action of $\mathscr{B}$, such that both actions commute. 
Definition 2.15. Let $V$ be a Hilbert $A$ - $B$-bimodule. The left multipliers of $V$ are

$$
L M(V)=\operatorname{Hom}_{-, B}(B, V),
$$

i.e., the $B$-linear homomorphisms from $B$ to $V$. The corresponding right multipliers are given by

$$
R M(V)=\operatorname{Hom}_{A,-}(A, V) .
$$

In particular $L M(A)$, where $A$ is considered as an $A$ - $A$-bimodule is a Banach algebra with multiplication given by composition of homomorphisms. In a similar way, we turn $R M(A)$ into a Banach algebra, but here we will use the opposite multiplication, i.e.,

$$
\alpha_{1} \cdot \alpha_{2}:=\alpha_{2} \circ \alpha_{1}
$$

for $\alpha_{i} \in R M(A)$. With this convention $A$ is a left ideal in $L M(A)$ and a right ideal in $R M(A)$.

Define $Q M(V):=\operatorname{Hom}_{A, B}(A \times B, V)$ as the set of bounded $(A, B)$ bilinear maps as in (1).

$Q M(V)$ comes equipped with an $A$ - $B$-bimodule structure in the following way. Let $a, a^{\prime} \in A, b, b^{\prime} \in B, q \in Q M(V)$, then

$$
(q \triangleright b)\left(a^{\prime}, b^{\prime}\right):=q\left(a^{\prime}, b b^{\prime}\right), \quad(a \triangleleft q)\left(a^{\prime}, b^{\prime}\right)=q\left(a^{\prime} a, b^{\prime}\right) .
$$

This can be extended to a Banach $R M(A)-L M(B)$-bimodule structure via

$$
(q \triangleright \beta)(a, b):=q(a, \beta(b)), \quad(\alpha \triangleleft q)(a, b)=q(\alpha(a), b) .
$$

for $\alpha \in R M(A), \beta \in L M(B)$.

Remark 2.16. Obviously, if $A$ is unital, then $Q M(V)=L M(V)$. If $B$ is unital, then $Q M(V)=R M(V)$. And if both $A$ and $B$ are unital, then $Q M(V)=$ $V$.

Define a locally convex quasi-strict topology (we will denote it by the abbreviation q.s.) on $\operatorname{Hom}_{A, B}(A \times B, V)$ by the family of semi-norms

$$
\left\{v_{a, b}: a \in A, b \in B\right\},
$$

where $v_{a, b}(q)=\|a \triangleleft q \triangleright b\|, q \in \operatorname{Hom}_{A, B}(A \times B, V)$, and define $X:=[V]_{\text {q.s. }}$ as the completion of $V$ with respect to the quasi-strict topology, restricted to $V$. Now consider a Cauchy net $\mathbf{x}=\left\{x_{i}\right\}$ in the topological space $(V, q . s$.$) . For any a \in A, b \in B$ the net $\left\{a x_{i} b\right\}$ converges to some vector $q_{\mathbf{x}}(a, b) \in V$. 
Proposition 2.17. The correspondence $\mathbf{x} \mapsto q_{\mathbf{x}}$ is a linear isometric map from $X$ onto $Q M(V)$. In the other words, quasi-multipliers of $V$ coincide with the completion of $V$ with respect to the quasi-strict topology.

Proof. Obviously, $q_{\mathbf{x}}$ is a bilinear map for any Cauchy net $\mathbf{x}=\left\{x_{i}\right\}$ of the space $(V, q . s$.$) . By the Banach-Steinhaus theorem the set of real numbers$ $\left\{\left\|x_{i}\right\|\right\}$ is bounded, say by a constant $C$. Then $\left\|q_{\mathbf{x}}(a, b)\right\| \leq C\|a\|\|b\|$, so $q_{\mathbf{x}}$ actually belongs to $Q M(V)$. Now let $q \in Q M(V)$ be given, choose approximate units $\left\{e_{\alpha}\right\}$ in $A$ and $\left\{u_{\beta}\right\}$ in $B$. Since

$$
(a \triangleleft q \triangleright b)\left(e_{\alpha}, u_{\beta}\right)=q\left(e_{\alpha} a, b u_{\beta}\right) \rightarrow q(a, b)
$$

for all $a \in A, b \in B$, the net $\mathbf{y}=\left\{q\left(e_{\alpha}, u_{\beta}\right)\right\}$ is a Cauchy net in $(V, q . s$.$) and$ $q=q_{\mathbf{y}}$, so $X=Q M(V)$ as required.

Consider also the locally convex strong topology (we will denote it by the abbreviation $s$ ) of point-wise convergence on $\operatorname{Hom}_{A, B}(A \times B, V)$ defined by the family of semi-norms

$$
\left\{\mu_{a, b}: a \in A, b \in B\right\}
$$

where $\mu_{a, b}(q)=\|q(a, b)\|, q \in \operatorname{Hom}_{A, B}(A \times B, V)$. Both these topologies - quasi-strict and strong - coincide on $V$ considered as a subspace of $Q M(V)$. This assertion may be strengthened in the following way.

LEMMA 2.18. $v_{a, b}(q)=\mu_{a, b}(q)$, i.e., $\|q(a, b)\|=\|a \triangleleft q \triangleright b\|$, for any $q \in Q M(V), a \in A, b \in B$.

Proof. Let $q \in Q M(V), a \in A, b \in B$ be given, choose approximate units $\left\{e_{\alpha}\right\}$ in $A$ and $\left\{u_{\beta}\right\}$ in $B$. Then the net $q\left(e_{\alpha} a, b u_{\beta}\right)=(a \triangleleft q \triangleright b)\left(e_{\alpha}, u_{\beta}\right)$ converges in norm to $q(a, b)$. It implies that $\|q(a, b)\|=\lim \| a \triangleleft q \triangleright$ $b\left(e_{\alpha}, u_{\beta}\right)\|\leq\| a \triangleleft q \triangleright b \|$. On the other hand,

$$
\begin{aligned}
\|a \triangleleft q \triangleright b\| & =\sup \{\|(a \triangleleft q \triangleright b)(c, d)\|:\|c\| \leq 1,\|d\| \leq 1, c \in A, d \in B\} \\
& =\sup \{\|q(c a, b d)\|:\|c\| \leq 1,\|d\| \leq 1, c \in A, d \in B\} \\
& =\sup \{\|c q(a, b) d\|:\|c\| \leq 1,\|d\| \leq 1, c \in A, d \in B\} \\
& \leq\|q(a, b)\|,
\end{aligned}
$$

which proves the inverse inequality.

Consider the canonical embedding $\Gamma: V \rightarrow Q M(V)$ given by (2). This way $Q M(V)$ provides an extension of $V$.

Definition 2.19. A quasi extension of a Hilbert $A$ - $B$-bimodule $V$ consists of: 
(i) two Banach algebras $\mathscr{A}$ and $\mathscr{B}$, such that $A \subset \mathscr{A}$ is a right ideal and $B \subset \mathscr{B}$ is a left ideal,

(ii) a Banach $\mathscr{A}$ - $\mathscr{B}$-bimodule $W$

(iii) and an isometric bimodule homomorphism $\Phi: V \longrightarrow W$ with

$$
\operatorname{Im}(\Phi)=A W B:=\overline{\operatorname{span}\{a x b: a \in A, x \in W, b \in B\}} .
$$

Definition 2.20. A quasi extension $(W, \mathscr{A}, \mathscr{B}, \Phi)$ of $V$ is said to be strictly essential if $A \subset \mathscr{A}$ is a right strictly essential ideal, i.e.,

$$
\|\alpha\|=\sup \{\|a \alpha\|: a \in A,\|a\| \leq 1\} \quad \text { for all } \quad \alpha \in \mathscr{A},
$$

$B \subset \mathscr{B}$ is a left strictly essential ideal, i.e.,

$$
\|\beta\|=\sup \{\|\beta b\|: b \in B,\|b\| \leq 1\} \quad \text { for all } \quad \beta \in \mathscr{B}
$$

and the following condition holds

(4)

$$
\|y\|=\sup \{\|a y b\|: a \in A, b \in B,\|a\| \leq 1,\|b\| \leq 1\} \quad \text { for all } \quad y \in W .
$$

Definition 2.21. A strictly essential quasi extension $(\widehat{W}, \widehat{\mathscr{A}}, \widehat{\mathscr{B}}, \widehat{\Phi})$ of $V$ is said to be maximal if for any other strictly essential quasi extension $(W, \mathscr{A}, \mathscr{B}, \Phi)$ there are an isometric homomorphism $\lambda: \mathscr{A} \rightarrow \widehat{A}$, which is the identity on $A$, an isometric homomorphism $\mu: \mathscr{B} \rightarrow \widehat{\mathscr{B}}$, which is the identity on $B$ and an isometric linear map $\Theta: W \rightarrow \widehat{W}$ such that it satisfies the condition

$$
\Theta(a y b)=\lambda(a) \Theta(y) \mu(b) \quad \text { for all } \quad a \in \mathscr{A}, y \in W, b \in \mathscr{B},
$$

and such that the diagram

is commutative.

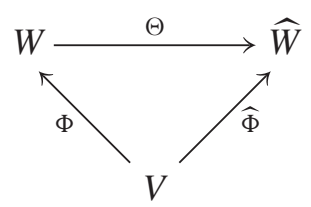

Theorem 2.22. Given an A-B-bimodule $V$. Then $(Q M(V), R M(A)$, $L M(B), \Gamma)$ is a maximal strictly essential quasi extension of $V$, where $\Gamma$ is defined by (2).

Proof. $A \subset R M(A)$ is a right strictly essential ideal and $B \subset L M(B)$ is a left strictly essential ideal by [13, Lemma 6]. Using approximate units of $A$ 
and $B$ a straightforward verification yields the formula (4). Now let us check the third condition of Definition 2.19. Obviously, $\operatorname{Im} \Gamma \subset A Q M(V) B$ and we only have to ensure the inverse inclusion. Given arbitrary $q \in Q M(V), a \in$ $A, b \in B$. Then for any $c \in A, d \in B$ one has

$$
(a \triangleleft q \triangleright b)(c, d)=q(c a, b d)=c q(a, b) d=\Gamma(q(a, b))(c, d) .
$$

Because $\Gamma$ is an isometry, $\operatorname{Im}(\Gamma)$ is closed, hence

$$
\operatorname{Im} \Gamma=A Q M(V) B
$$

and $(Q M(V), R M(A), \operatorname{LM}(B), \Gamma)$ is a strictly essential quasi extension of $V$. To establish its maximality one chooses any other strictly essential quasi extension $(W, \mathscr{A}, \mathscr{B}, \Phi)$ of $V$. By [13] $L M(B)$ is a maximal left strictly essential extension of $B$ and, consequently, there is an isometric homomorphism $\mu: \mathscr{B} \rightarrow \operatorname{LM}(B)$, which restricts to the identity on $B$. Similarly, there is an isometric homomorphism $\lambda: \mathscr{A} \rightarrow R M(A)$, which acts identically on $A$. Now for $y \in W, a \in A, b \in B$ put

$$
\Xi(y)(a, b)=\Phi^{-1}(a y b) .
$$

Obviously, $\Xi(y)$ is a bilinear map from $A \times B$ to $V$. Moreover, $\Xi$ is actually an isometry, because

$$
\begin{aligned}
\|\Xi(y)\| & =\sup \left\{\left\|\Phi^{-1}(a y b)\right\|: a \in A, b \in B,\|a\| \leq 1,\|b\| \leq 1\right\} \\
& =\sup \{\|a y b\|: a \in A, b \in B,\|a\| \leq 1,\|b\| \leq 1\} \\
& =\|y\|,
\end{aligned}
$$

where we have used item (iii) of Definition 2.19 and condition (4). Now choose $a \in A, \alpha \in \mathscr{A}, b \in B, \beta \in \mathscr{B}$ and $y \in W$. On the one hand one has

$$
\Xi(\alpha y \beta)(a, b)=\Phi^{-1}(a \alpha y \beta b)
$$

and on the other hand

$$
\begin{aligned}
(\lambda(\alpha) \triangleleft \Xi(y) \triangleright \mu(\beta))(a, b) & =\Xi(y)(\lambda(\alpha)(a), \mu(\beta)(b)) \\
& =\Phi^{-1}([\lambda(\alpha)(a)] y[\mu(\beta)(b)]) \\
& =\Phi^{-1}(a \alpha y \beta b) .
\end{aligned}
$$

So, the map $\Xi$ satisfies the condition (5). The theorem is proved. 


\section{Quasi-multipliers of Hilbert $C^{*}$-bimodules in Kasparov's sense}

Let us begin by recalling the definition of Hilbert $C^{*}$-bimodules in Kasparov's sense, which is the starting point for $K K$-theory (cf., e.g., [10]). Given two $C^{*}$-algebras $A$ and $B$, one considers a right $\mathrm{Z} / 2 \mathrm{Z}$-graded Hilbert $B$-module $V$ and a $*$-homomorphism $\rho: A \rightarrow \operatorname{End}_{B}^{*}(V)^{(0)}$, where $\operatorname{End}_{B}^{*}(V)^{(0)}$ denotes the 0 -homogeneous adjointable operators in $V$. We will additionally assume that this representation is faithful and non-degenerate. Then, in particular, the $C^{*}$-algebra $\rho(A)$ is isomorphic to $A$, and its left action on $V$ is given by the formula

$$
a \triangleleft x=\rho(a)(x), \quad a \in A, x \in V .
$$

The right action of $B$ on $V$ will sometimes be denoted by

$$
x \triangleright b=x b, \quad b \in B, x \in V .
$$

Let us remark that, in fact, we may restrict our considerations concerning (left, right or quasi) multipliers of $V$ to the non-graded case, because $\operatorname{End}_{B}^{*}(V)^{(0)}=$ $\operatorname{End}_{B}^{*}\left(V_{1}\right) \oplus \operatorname{End}_{B}^{*}\left(V_{2}\right)$, where $V=V_{1} \oplus V_{2}$ means the given Z/2Z-graduation of $V$. So henceforth we assume that the module $V$ is non-graded and the (faithful, non-degenerate) representation $\rho$ is of the form $\rho: A \rightarrow \operatorname{End}_{B}^{*}(V)$.

Definition 3.1. Quasi-multipliers $Q M_{(A, \rho, B)}(V)$ of $V$ are defined as the set of all bimodule homomorphisms from $A \times B$ to $V$, i.e.,

$$
Q M_{(A, \rho, B)}(V)=\operatorname{Hom}_{A, B}(A \times B, V) .
$$

The Banach space of quasi-multipliers $Q M_{(A, \rho, B)}(V)$ carries an $R M(A)$ $L M(B)$-bimodule structure via

$$
(q \triangleright \beta)(a, b)=q(a, \beta(b)), \quad(\alpha \triangleleft q)(a, b)=q(\alpha(a), b)
$$

for $\alpha \in R M(A), \beta \in L M(B)$.

Proposition 3.2. $V$ is isometrically embedded into $Q M_{(A, \rho, B)}(V)$ by the bimodule map

$$
\begin{aligned}
& \Gamma_{(A, \rho, B)}: V \rightarrow Q M_{(A, \rho, B)}(V), \\
& \Gamma_{(A, \rho, B)}(x)(a, b):=a \triangleleft x \triangleright b:=\rho(a)(x b) .
\end{aligned}
$$

Proof. Given $x \in V, a, a^{\prime} \in A, b, b^{\prime} \in B$. Denote the quasi-multiplier $\Gamma_{(A, \rho, B)}(x)$ by $q_{x}$ for brevity. Then

$$
\begin{aligned}
q_{a^{\prime} \triangleleft x \triangleright b^{\prime}}(a, b) & =\rho(a)\left(\rho\left(a^{\prime}\right)\left(x b^{\prime}\right) b\right) \\
& =q_{x}\left(\rho(a) \rho\left(a^{\prime}\right), b^{\prime} b\right) \\
& =\left(a^{\prime} \triangleleft q_{x} \triangleright b^{\prime}\right)(a, b),
\end{aligned}
$$


so $\Gamma_{(A, \rho, B)}$ is a bimodule map and it only remains to check that it is an isometry. Then

$$
\left\|q_{x}\right\|=\sup \{\|\rho(a)(x b)\|:\|a\| \leq 1,\|b\| \leq 1, a \in A, b \in B\} \leq\|x\|
$$

and we have to show that this supremum achieves the value $\|x\|$. For this it is enough to verify that

$$
\|x\|=\sup \{\|\rho(a)(x)\|:\|a\| \leq 1, a \in A\} .
$$

Because the representation $\rho$ is non-degenerate, the sub-bimodule $W=$ $\operatorname{span}\{\rho(a)(x): a \in A, x \in V\}$ is dense in $V$ and, consequently, we need to prove (7) only for the vectors $x \in W$. So, choose an arbitrary $x \in W$, i.e., $x=\sum \rho\left(a_{i}\right) y_{i}$ with $y_{i} \in V$. Let $\left\{e_{\alpha}\right\}$ be an approximate unit of $A$. Then $\rho\left(e_{\alpha}\right) x=\sum \rho\left(e_{\alpha} a_{i}\right) y_{i}$ converges to $x$, and the supremum in (7) achieves the norm $\|x\|$ on the approximate unit $\left\{\rho\left(e_{\alpha}\right)\right\}$ of $\rho(A)$.

In fact, we may carry out these considerations even for the category of Banach bimodules over $C^{*}$-algebras, which act non-degenerately. More precisely, given two $C^{*}$-algebras $A$ and $B$ and a Banach $A$ - $B$-bimodule $X$ such that the following conditions hold

$$
\overline{\operatorname{span}\{a x: a \in A, x \in X\}}=X
$$

and

$$
\overline{\operatorname{span}\{x b: b \in B, x \in X\}}=X \text {. }
$$

Then quasi-multipliers $Q M(X)$ of $X$ are defined again as the set $\operatorname{Hom}_{A, B}(A \times$ $B, X)$.

LemMa 3.3. The two conditions (8) and (9) are equivalent to the following one

$$
\overline{\operatorname{span}\{a x b: a \in A, b \in B, x \in X\}}=X .
$$

Proof. Let $X$ satisfy both (8) and (9) and let an arbitrary $y \in X$ and $\varepsilon>0$ be given. There are $a_{i} \in A, x_{i} \in X$ such that

$$
\left\|y-\sum_{i=1}^{n} a_{i} x_{i}\right\|<\varepsilon
$$

and for any $i$ there are $b_{j}^{(i)} \in B, z_{j}^{(i)} \in X$ such that

$$
\left\|x_{i}-\sum_{j=1}^{m_{i}} z_{j}^{(i)} b_{j}^{(i)}\right\|<\frac{\varepsilon}{\left\|a_{i}\right\| n} .
$$


Then

$\left\|y-\sum_{i=1}^{n} \sum_{j=1}^{m_{i}} a_{i} z_{j}^{(i)} b_{j}^{(i)}\right\| \leq\left\|y-\sum_{i=1}^{n} a_{i} x_{i}\right\|+\left\|\sum_{i=1}^{n} a_{i} x_{i}-\sum_{i=1}^{n} \sum_{j=1}^{m_{i}} a_{i} z_{j}^{(i)} b_{j}^{(i)}\right\|<2 \varepsilon$.

The inverse implication of the lemma is trivial.

Proposition 3.4. X is isometrically embedded into $Q M(X)$ by the bimodule map

$$
\Gamma: X \rightarrow Q M(X), \quad \Gamma(x)(a, b)=a x b .
$$

Proof. We only have to check that for any $x \in X$ one has

$$
\|x\|=\sup \{\|a x b\|:\|a\| \leq 1,\|b\| \leq 1, a \in A, b \in B\} .
$$

By Lemma 3.3 the vector $x$ may be approximated in norm by vectors of the form $\sum c_{i} y_{i} d_{i}$ with $c_{i} \in A, y_{i} \in X, d_{i} \in B$. Then

$$
\left\|\sum c_{i} y_{i} d_{i}\right\|=\sup \left\{\left\|e_{\alpha} \sum c_{i} y_{i} d_{i} u_{\beta}\right\|: \alpha, \beta\right\},
$$

where $\left\{e_{\alpha}\right\}$ and $\left\{u_{\beta}\right\}$ stand for approximate units in $A$ and $B$ respectively.

\section{Quasi-multipliers of direct sums of bimodules}

Given two $C^{*}$-algebras $A$ and $B$ and a Hilbert $A$ - $B$-bimodule $V$. Consider another $A$ - $B$-bimodule $\widetilde{V}$ and a bimodule homomorphism $\theta: V \rightarrow \widetilde{V}$. Then there is a homomorphism $\theta_{*}: Q M(V) \rightarrow Q M(\widetilde{V})$ of Banach $R M(A)-L M(B)$ bimodules given by the formula

$$
\theta_{*}(q)=\theta q, \quad q \in Q M(V) .
$$

So, quasi-multipliers provide a covariant functor $Q M$ from the category of Hilbert $A$ - $B$-bimodules to the category of Banach $R M(A)-L M(B)$-bimodules. Obviously, these observations are still valid for Banach (instead of Hilbert) $A$ $B$-bimodules, on which both $C^{*}$-algebras $A$ and $B$ act non-degenerately. If $V$ is given as a direct sum $V=V_{1} \oplus V_{2}$ of its (closed) sub-bimodules $V_{1}$ and $V_{2}$, then one straightforwardly verifies that $Q M(V)=Q M\left(V_{1}\right) \oplus Q M\left(V_{2}\right)$, in other words the functor $Q M$ is additive. In particular, for the free $A$ - $A$-bimodule $A^{n}$ one has $Q M\left(A^{n}\right)=Q M(A)^{n}$.

Now we are investigating what happens with quasi-multipliers if we map either $A$ or $B$ to other $C^{*}$-algebras. So, consider two $C^{*}$-algebras $\widetilde{A}$ and $\widetilde{B}$ and two surjective $*$-homomorphisms

$$
\varphi: A \rightarrow \widetilde{A}, \quad \psi: B \rightarrow \widetilde{B} .
$$


Assume $V$ is a Banach $\widetilde{A}$ - $\widetilde{B}$-bimodule equipped with non-degenerate actions of these $C^{*}$-algebras. Define a left action $\triangleleft_{\varphi}$ of $A$ twisted by $\varphi$ and right action $\triangleright_{\psi}$ of $B$ twisted by $\psi$ on $V$ as follows

$$
a \triangleleft_{\varphi} x=\varphi(a) \triangleleft x, \quad x \triangleright_{\psi} b=x \triangleright \psi(b),
$$

where $a \in A, b \in B, x \in V$. Surjectivity of $\varphi$ and $\psi$ ensures that these actions are non-degenerate. Then $\left(V, \triangleleft_{\varphi}, \triangleright_{\psi}\right)$ is a Banach $A$ - $B$-bimodule and quasimultipliers of this bimodule are called twisted quasi-multipliers of the original $\tilde{A}-\tilde{B}$-bimodule $(V, \triangleleft, \triangleright)$ and are denoted by $Q M_{(\varphi, \psi)}(V)$.

With this construction, quasi-multipliers are contravariant in both variables $A$ and $B$.

Now we are going to study the behavior of the functor $Q M$ with respect to infinite direct sums of bimodules. As a corollary, in particular, we will obtain a description of quasi-multipliers for the standard $A$-A-bimodule $l_{2}(A)$. So given $A$-B-bimodules $V_{i}$. Obviously, for a sequence $\left(x_{i}\right), x_{i} \in V_{i}$ the series $\sum_{i}{ }_{A}\left\langle x_{i}, x_{i}\right\rangle$ converges in norm if and only if the series $\sum_{i}\left\langle x_{i}, x_{i}\right\rangle_{B}$ does, moreover, their norms have to coincide. Set

$$
V=\left\{\left(x_{i}\right): x_{i} \in V_{i}, \sum_{i}\left\langle x_{i}, x_{i}\right\rangle \text { converges in norm }\right\} .
$$

Then $V$ is a Hilbert $A$ - $B$-bimodule with respect to the inner products

$$
{ }_{A}\langle x, y\rangle=\sum_{i}\left\langle x_{i}, y_{i}\right\rangle \quad \text { and } \quad\langle x, y\rangle_{B}=\sum_{i}\left\langle x_{i}, y_{i}\right\rangle_{B},
$$

where $x=\left(x_{i}\right), y=\left(y_{i}\right) \in V$ (cf. [12, Example 1.3.5]).

THEOREM 4.1. Set

$$
\begin{aligned}
& W=\left\{\left(q_{i}\right): q_{i} \in Q M\left(V_{i}\right),\right. \text { the norms of the operators } \\
& \rho_{n}=\left(q_{1}, \ldots, q_{n}, 0, \ldots\right): A \times B \rightarrow \bigoplus_{i=1}^{n} V_{i} \text { are uniformly bounded } \\
& \text { over } \left.n \text {, and }\left(q_{i}(a, b)\right) \in V \text { for any } a \in A, b \in B\right\} .
\end{aligned}
$$

In particular, if $\left(q_{i}\right) \in W$ then both series $\sum_{i}\left\langle q_{i}(a, b), q_{i}(a, b)\right\rangle$ and $\sum_{i}\left\langle q_{i}(a, b), q_{i}(a, b)\right\rangle_{B}$ converge in norm for any $a \in A, b \in B$. Then $W$, with norm defined by (10) below, is a Banach RM(A)-LM(B)-bimodule with entry-wise action, isometrically isomorphic to the Banach $R M(A)-L M(B)$ bimodule $Q M(V)$. 
Proof. Suppose $r \in R M(A), l \in L M(B)$ and $q=\left(q_{i}\right) \in W$. Then

$$
\begin{aligned}
\sum_{i} A_{\langle}\left\langle\left(r \triangleleft q_{i} \triangleright l\right)(a, b),\left(r \triangleleft q_{i}\right.\right. & \triangleright l)(a, b)\rangle \\
& =\sum_{i}{ }_{A}\left\langle q_{i}(r(a), l(b)), q_{i}(r(a), l(b))\right\rangle
\end{aligned}
$$

and $r \triangleleft q \triangleright l:=\left(r \triangleleft q_{i} \triangleright l\right)$ belongs to $W$. Set

$$
\|q(a, b)\|:=\left\|\sum_{i}{ }_{A}\left\langle q_{i}(a, b), q_{i}(a, b)\right\rangle\right\|^{1 / 2}
$$

and

$$
\|q\|:=\sup \{\|q(a, b)\|:\|a\| \leq 1,\|b\| \leq 1, a \in A, b \in B\} .
$$

This supremum is finite, because $q$ is a point-wise limit of the sequence

$$
\left\{\rho_{n}=\left(q_{1}, \ldots, q_{n}, 0, \ldots\right)\right\}
$$

and $\left\|\rho_{n}\right\| \leq C$ for any $n$. Thus, $W$ is a normed $R M(A)-L M(B)$-bimodule. Note, moreover, that $q$ considered as a map $q: A \times B \rightarrow V$ is bounded and thus a quasi-multiplier.

An isometric isomorphism $\Phi: Q M(V) \rightarrow W$ may be defined in the following way. Denote by $p_{i}: V \rightarrow V_{i}$ the natural projection and consider any quasimultiplier $T \in Q M(V)$, i.e., $T: A \times B \rightarrow V$. Then, clearly, $T_{i}=p_{i} T$ belongs to $Q M\left(V_{i}\right)$ for any $i$ and the sequence $\left\{F_{n}=\left(T_{1}, \ldots, T_{n}, 0, \ldots\right)\right\} \subset Q M(V)$ quasi-strictly converges to $T$. By definition set $\Phi(T)=\left(T_{i}\right)$.

Because $T(a, b)=\left(T_{1}(a, b), T_{2}(a, b), \ldots\right) \in \bigoplus V_{i}$ for any $a \in A, b \in B$, the sequence $\left(T_{i}\right)$ belongs to $W$. Obviously, $\Phi$ is an isometry. Now take an arbitrary $\left(q_{i}\right) \in W$. Define $T(a, b):=\left(q_{1}(a, b), q_{2}(a, b), \ldots\right)$ for $a \in A, b \in$ $B$. Then $T$ is an element of $Q M(V)$ and $\Phi(T)=\left(q_{i}\right)$, proving surjectivity of $\Phi$.

COROLlaRY 4.2. Quasi-multipliers of the standard bimodule $l_{2}(A)$ over a $C^{*}$-algebra A coincide with the set of sequences $\left\{\left(q_{i}\right), q_{i} \in Q M(A)\right\}$ such that the norms of $\left\{\bigoplus_{i=1}^{n} q_{i}\right\}$ are uniformly bounded over $n$ and $\sum_{i}\left(a q_{i} c\right)^{*}\left(a q_{i} c\right)$ converges in norm for any a, $c \in A$.

Let $V$ be a right Hilbert module over a $C^{*}$-algebra $B$. Then its multipliers were defined in [2], [16] as $\operatorname{Hom}_{B}^{*}(B, V)$. It is a Hilbert module over the $C^{*}$-algebra $M(B)$. Likewise, the left multipliers of $V$ were defined in [8] as $\operatorname{Hom}_{B}(B, V)$ being a Banach module over the Banach algebra $L M(B)$. The arguments above imply the following assertion. 
THEOREM 4.3. Assume that $V=\bigoplus V_{i}$ is a direct sum of Hilbert B-modules $V_{i}$. Then

$$
\begin{gathered}
L M(V)=\left\{\left(\lambda_{i}\right): \lambda_{i} \in L M\left(V_{i}\right),\right. \text { the norms of the operators } \\
\theta_{n}=\left(\lambda_{1}, \ldots, \lambda_{n}, 0, \ldots\right): B \rightarrow \bigoplus_{i=1}^{n} V_{i} \text { are uniformly bounded } \\
\text { over } \left.n \text {, and }\left(\lambda_{i}(b)\right) \in V \text { for any } b \in B\right\}, \\
M(V)=\left\{\left(\mu_{i}\right): \mu_{i} \in M\left(V_{i}\right),\right. \text { the norms of the operators } \\
\tau_{n}=\left(\mu_{1}, \ldots, \mu_{n}, 0, \ldots\right): B \rightarrow \bigoplus_{i=1}^{n} V_{i} \text { are uniformly bounded } \\
\text { over } \left.n \text {, and }\left(\mu_{i}(b)\right) \in V \text { for any } b \in B\right\} .
\end{gathered}
$$

This theorem in its part concerning multipliers generalizes [2, Theorem 2.1], where the crucial case of the standard module was considered. Our description being applied to $V=l_{2}(A)$ differs from the one of [2], but is just its equivalent reformulation. Indeed, let $V=l_{2}(A), m_{i} \in M(A)$ and the sequence $\left\{\tau_{n}=\right.$ $\left.\left(m_{1}, \ldots, m_{n}, 0, \ldots\right)\right\}$ be given. Then one has

$$
\begin{aligned}
\left\|\tau_{n}\right\|^{2} & =\sup \left\{\left\|\left\langle\tau_{n}(a), \tau_{n}(a)\right\rangle\right\|: a \in A,\|a\| \leq 1\right\} \\
& =\sup \left\{\left\|\sum_{i=1}^{n} m_{i}(a)^{*} m_{i}(a)\right\|: a \in A,\|a\| \leq 1\right\} \\
& =\sup \left\{\left\|\sum_{i=1}^{n} a^{*} m_{i}^{*} m_{i} a\right\|: a \in A,\|a\| \leq 1\right\} \\
& =\left\|\sum_{i=1}^{n} m_{i}^{*} m_{i}\right\| .
\end{aligned}
$$

Now, [2, Theorem 2.1] claims that

$$
\begin{aligned}
M\left(l_{2}(A)\right)=\left\{\left(m_{n}\right): m_{n} \in M(A),\right. & \\
& \left.\sum a m_{n}^{*} m_{n}, \sum m_{n}^{*} m_{n} a \text { converge in } A \text { for any } a \in A\right\} .
\end{aligned}
$$

But the norm-convergence of a series $\sum a^{*} m_{n}^{*} m_{n} a$ and uniform boundedness of the sequence $\left\{\left\|\sum m_{n}^{*} m_{n}\right\|\right\}$ (say by a constant $C$ ), which is ensured by the equality (11), imply the norm convergence of the series $\sum a x_{n}^{*} x_{n}$ and $\sum x_{n}^{*} x_{n} a$ 
because of the Cauchy-Schwartz inequality

$$
\begin{aligned}
\left\|\sum m_{n}^{*} m_{n} a\right\| & \leq\left\|\sum m_{n}^{*} m_{n}\right\|^{1 / 2} \cdot\left\|\sum a^{*} m_{n}^{*} m_{n} a\right\|^{1 / 2} \\
& \leq\left\|\sum a^{*} m_{n}^{*} m_{n} a\right\|^{1 / 2} C^{1 / 2} .
\end{aligned}
$$

\section{Quasi-multipliers of continuous sections of Hilbert $C^{*}$-bimodule bundles}

Given a locally compact Hausdorff space $X$. For the commutative $C^{*}$-algebra $C_{0}(X)$ of continuous functions on $X$ vanishing at infinity its set of multipliers (as well as its set of left (or right) multipliers and quasi-multipliers) coincides with the $C^{*}$-algebra $C_{b}(X)$ of bounded continuous functions on $X$. On the other hand $C_{b}(X)$ is nothing else but the $C^{*}$-algebra $C(\beta X)$ of continuous functions on the Stone-Čech compactification of $X$ (cf. $[15,3.12 .6])$. This result was extended in [1] to $C^{*}$-algebras $A_{0}(X)=C_{0}(X, A)$ of continuous $A$-valued functions vanishing at infinity, where $A$ is a $C^{*}$-algebra (actually in [1] there was considered the even more general case of continuous cross sections of fiber spaces). Denote by $M(A)_{\beta}$ the $C^{*}$-algebra of multipliers of $A$, equipped with the strict topology, and by $C_{b}\left(X, M(A)_{\beta}\right)$ the set of continuous bounded $M(A)$-valued functions on $X$. Then

$$
M\left(A_{0}(X)\right)=C_{b}\left(X, M(A)_{\beta}\right)
$$

(cf. [1, Corollary 3.4]). But $C_{b}\left(X, M(A)_{\beta}\right)$ is not isomorphic to $C(\beta X$, $\left.M(A)_{\beta}\right)$, because $C(\beta X) \otimes M(A)=M\left(C_{0}(X)\right) \otimes M(A) \varsubsetneqq M\left(C_{0}(X) \otimes A\right)=$ $M\left(A_{0}(X)\right)$ whenever $X$ is $\sigma$-compact, $A$ is infinite dimensional and the tensor products are considered with respect to the minimal (spatial) norm, [1, Theorem 3.8].

And in turn formula (12) was extended in [5] in the following way. Let $V$ be a Hilbert $A$-module and $V_{0}(X)=C_{0}(X, V)$ be the set of continuous $V$-valued functions vanishing at infinity. It is, obviously, a Hilbert $A_{0}(X)$-module. Denote by $\operatorname{End}_{A}^{*}(V)_{\beta}$ the $C^{*}$-algebra of all $A$-linear bounded adjointable operators in $V$, equipped with the $*$-strict module topology (cf. [12, § 5.5]). Then

$$
\operatorname{End}_{A_{0}(X)}^{*}\left(V_{0}(X)\right)=C_{b}\left(X, \operatorname{End}_{A}^{*}(V)_{\beta}\right) .
$$

Because by Kasparov's theorem $\operatorname{End}_{A}^{*}(V)=M\left(K_{A}(V)\right)$ (cf. [9]) for any Hilbert $A$-module $V$, where $K_{A}(V)$ stands for the $C^{*}$-algebra of compact operators of $V$, the formula (12) is a particular case of (13) for $V=A$. Our aim in this paragraph is to find the proper analogue of formula (12) for quasimultipliers of continuous sections of Hilbert $C^{*}$-bimodule bundles. 
In order to define this notion, take a locally compact Hausdorff space $X$ and two $C^{*}$-algebras $A$ and $B$, set $A_{0}(X):=C_{0}(X, A)$ and $B_{0}(X):=C_{0}(X, B)$. Equipped with the supremum norm, these are again $C^{*}$-algebras.

In view of the above observations we want sections in our still to be defined bundles of Hilbert $A$ - $B$-bimodules to form a Hilbert $A_{0}(X)$ - $B_{0}(X)$-bimodule with the inner product induced by the pointwise operations in the fibers. The corresponding structure group should therefore reduce to unitary $A$ - $B$-linear operators, which raises the question whether these are well-defined, since we have two inner products. This is settled by the following lemma.

Lemma 5.1. Let $V$ be a Hilbert $A$-B-bimodule and $T \in \operatorname{End}_{A, B}(V)$ be a bounded $A$-and B-linear operator, which has an adjoint $T^{*, B}$ for the $B$-valued inner product. Then $T^{*, B}$ coincides with the adjoint of $T$ for the A-valued inner product (i.e., $T^{*, A}=T^{*, B}$ ).

Proof. We follow [3, Remark 1.9]. Let $x, y, z \in V$, then we have

$$
\begin{aligned}
{ }_{A}\left\langle x, T^{*, B} y\right\rangle z & =x\left\langle T^{*, B} y, z\right\rangle_{B}=x\langle y, T z\rangle_{B}={ }_{A}\langle x, y\rangle T z=T\left({ }_{A}\langle x, y\rangle z\right) \\
& =T\left(x\langle y, z\rangle_{B}\right)=T x\langle y, z\rangle_{B}={ }_{A}\langle T x, y\rangle z
\end{aligned}
$$

Clearly $a={ }_{A}\left\langle x, T^{*, B} y\right\rangle-{ }_{A}\langle T x, y\rangle \in{ }_{A}\langle V, V\rangle$, where the latter denotes the closure of the linear span of all possible $A$-valued inner products. Moreover $a z=0$ for all $z \in V$ by the previous calculation. This implies $a=0$ by the approximate unit argument given in [3, Remark 1.9].

Definition 5.2. Let $V$ be a Hilbert $A$ - $B$-bimodule. By the above lemma, the adjointable $A$ - $B$-linear operators $\operatorname{End}_{A, B}^{*}(V)$ are well-defined. Denote the unitary elements in this $C^{*}$-algebra by $U_{A, B}(V)$.

Definition 5.3. Given a locally compact Hausdorff space $X$ and a Hilbert $A$ - $B$-bimodule $V$. A Hilbert $A$ - $B$-bimodule bundle $\mathscr{V}$ over $X$ with typical fiber $V$ is a triple $(\mathscr{V}, p, X)$, where $\mathscr{V}$ is a Hausdorff space and $p: \mathscr{V} \rightarrow X$ maps $\mathscr{V}$ onto $X$ such that the following holds:

(i) there is an open cover $\left\{U_{i}\right\}_{i \in I}$ of $X$ such that there exist homeomorphisms

$$
\varphi_{i}: p^{-1}\left(U_{i}\right) \longrightarrow U_{i} \times V
$$

with $\operatorname{pr}_{1} \circ \varphi_{i}=\left.p\right|_{p^{-1}\left(U_{i}\right)}$.

(ii) let $\bar{\varphi}_{i j}$ be defined via $\varphi_{j} \circ \varphi_{i}^{-1}(x, v)=\left(x, \bar{\varphi}_{i j}(x)(v)\right)$ for $x \in U_{i} \cap U_{j}$ and $v \in V$, then $\bar{\varphi}_{i j}$ is a continuous map

$$
\bar{\varphi}_{i j}: U_{i} \cap U_{j} \longrightarrow U_{A, B}(V) .
$$


Condition (i) implies that $\mathscr{V}$ is fiberwise isomorphic to $V$, condition (ii) encodes the reduction of the structure group to the unitary operators. The continuous sections $\mathscr{V}_{0}(X)=C_{0}(X, \mathscr{V})$ indeed yield a $A_{0}(X)$ - $B_{0}(X)$-bimodule. Let $x \in X$ be in the set $U_{i}$ of the cover, then there is an $A_{0}(X)$-valued inner product on $\mathscr{V}_{0}(X)$ defined via

$$
A_{0}(X)\langle\sigma, \tau\rangle(x)={ }_{A}\left\langle\mathrm{pr}_{2} \circ \varphi_{i} \circ \sigma(x), \mathrm{pr}_{2} \circ \varphi_{i} \circ \tau(x)\right\rangle,
$$

where $\mathrm{pr}_{2}$ stands for the projection of $U_{i} \times V$ onto $V$. This does not depend on the particular choice of $\left(U_{i}, \varphi_{i}\right)$. Indeed, if $x$ lies in $U_{i} \cap U_{j}$ we have:

$$
\begin{aligned}
{ }_{A}\left\langle\mathrm{pr}_{2} \circ \varphi_{i} \circ \sigma(x), \mathrm{pr}_{2} \circ \varphi_{i} \circ \tau(x)\right\rangle \\
\quad={ }_{A}\left\langle\bar{\varphi}_{j i}(x)\left(p r_{2} \circ \varphi_{j} \circ \sigma(x)\right), \bar{\varphi}_{j i}(x)\left(p r_{2} \circ \varphi_{j} \circ \tau(x)\right)\right\rangle \\
\quad={ }_{A}\left\langle\mathrm{pr}_{2} \circ \varphi_{j} \circ \sigma(x), \mathrm{pr}_{2} \circ \varphi_{j} \circ \tau(x)\right\rangle
\end{aligned}
$$

due to the unitarity of the structure group. There is a similar $B_{0}(X)$-valued inner product on $\mathscr{V}_{0}(X)$. With these additional structures $\mathscr{V}_{0}(X)$ is indeed an $A_{0}(X)$ - $B_{0}(X)$-bimodule.

Associated to $\mathscr{V}$ we have the bundle of quasi-multipliers $Q M(\mathscr{V})$. To define this, note that for a unitary $u \in U_{A, B}(V)$ and a quasi-multiplier $q \in Q M(V)$ the map $u \circ q$ is again a quasi-multiplier due to the $A-B$-linearity of $u$. Therefore the space

$$
\coprod_{i \in I} U_{i} \times Q M(V)
$$

may be equipped with the equivalence relation

$$
(x, q) \sim\left(x, \bar{\varphi}_{i j}(x) \circ q\right),
$$

where $i, j \in I, x \in U_{i j}$ and $q \in Q M(V)$. The quotient $Q M(\mathscr{V})=\bigsqcup_{i \in I} U_{i} \times$ $Q M(V) / \sim$ is a locally trivial bundle with typical fiber $Q M(V)$. Moreover the canonical map $\iota: V \rightarrow Q M(V)$ extends to a bundle morphism

$$
\mathscr{V} \longrightarrow Q M(\mathscr{V}) ; \quad v \mapsto\left[x, \iota \circ \operatorname{pr}_{2} \circ \varphi_{i}(v)\right],
$$

where $v$ belongs to the fiber over $x \in X$ and $[x, q] \in Q M(\mathscr{V})$ denotes the equivalence class of $(x, q)$. We may consider the quasi-strict topology on $Q M(V)$, the quotient topology induced by this on the space $Q M(\mathscr{V})$ will again be called the quasi-strict topology on the bundle $Q M(\mathscr{V})$. This is the last ingredient to phrase the analogue of (12) in the case of bundles.

THEOREM 5.4. For the quasi-multipliers of $\mathscr{V}_{0}(X)$ we have an isometric bimodule isomorphism

$$
Q M\left(\mathscr{V}_{0}(X)\right) \cong C_{b}(X, Q M(\mathscr{V})),
$$


where $Q M(\mathscr{V})$ on the right-hand side is equipped with the quasi-strict topology.

Proof. We are going to construct explicit maps in both directions and show that they are inverse to each other. Denote by $\pi: Q M(\mathscr{V}) \rightarrow X$ the bundle projection. For the map from the left hand side to the right we need an evaluation map turning a quasi-multiplier on sections $Q M\left(\mathscr{V}_{0}(X)\right)$ into a quasi-multiplier on a fixed fiber $Q M(\mathscr{V})_{y}=\pi^{-1}(y)$. Therefore we need to be able to construct sections of $A_{0}(X), B_{0}(X)$ with a prescribed value at a given point $y \in X$. Local compactness enables us to achieve this. Let $a \in A, b \in B$ be given. By passing to the one-point compactification $X^{+}$(which is normal) we can construct a function

$$
\chi^{y}: X^{+} \longrightarrow[0,1]
$$

which is 1 at $y$ and vanishes at $\infty$. In particular, we may set $\alpha=\chi^{y} a \in A_{0}(X)$ and $\beta=\chi^{y} b \in B_{0}(X)$.

If $\mathscr{V}_{y}$ denotes the fiber of $\mathscr{V}$ over $y \in X$, then $Q M(\mathscr{V})_{y}$ is by construction canonically isomorphic to $Q M\left(\mathscr{V}_{y}\right)$. Let $\alpha, \beta$ be sections of $A_{0}(X), B_{0}(X)$ as above and set

$$
\varphi_{y}: Q M\left(\mathscr{V}_{0}(X)\right) \longrightarrow Q M(\mathscr{V})_{y} ; \quad \varphi_{y}(G)(a, b)=G(\alpha, \beta)(y)
$$

To see that this does not depend on the choice of $\alpha$ note that $G(\cdot, \beta): A_{0}(X) \rightarrow$ $\mathscr{V}_{0}(X)$ is left $A_{0}(X)$-linear and bounded for any $\beta \in B_{0}(X)$, therefore

$$
A_{0}(X)\langle G(\alpha, \beta), G(\alpha, \beta)\rangle \leq\|G(\cdot, \beta)\|^{2} \cdot{ }_{A_{0}(X)}\langle\alpha, \alpha\rangle .
$$

If $\alpha(y)=0$ this implies $A_{0}(X)\langle G(\alpha, \beta), G(\alpha, \beta)\rangle(y)=0$. Thus, $\varphi_{y}$ does not depend on the choice of $\alpha$. The same argument shows that different choices of $\beta$ will lead to the same map $\varphi_{y}$. Furthermore

$$
\left\|\varphi_{y}(G)(a, b)\right\|=\|G(\alpha, \beta)(y)\| \leq\|G\|\|\alpha\|\|\beta\|=\|G\|\|a\|\|b\|
$$

proves that $\varphi_{y}(G)$ is bounded and therefore indeed defines an element of $Q M\left(\mathscr{V}_{y}\right)=Q M(\mathscr{V})_{y}$. Note that the upper bound can be chosen independently of $y \in X$.

Recall that a section $\sigma: X \rightarrow Q M(\mathscr{V})$ is continuous at $y \in Y$ if and only if there exists a trivialization $\psi_{U}:\left.Q M(\mathscr{V})\right|_{U} \rightarrow U \times Q M(V)$ such that the map $\left.\operatorname{pr}_{2} \circ \psi_{U} \circ \sigma\right|_{U}: U \rightarrow Q M(V)$ is continuous. Let $\phi_{U}:\left.\mathscr{V}\right|_{U} \rightarrow U \times V$ be a local trivialization of $\mathscr{V}$. By construction of $Q M(\mathscr{V})$ there is a corresponding trivialization $\psi_{U}$ such that for $y \in U, q \in Q M(\mathscr{V})_{y}=Q M\left(\mathscr{V}_{y}\right), a \in A$ and $b \in B$ we have

$$
\left(\operatorname{pr}_{2} \circ \psi_{U}(q)\right)(a, b)=\operatorname{pr}_{2} \circ \phi_{U}(q(a, b)) .
$$


Now let $\varepsilon>0$. Since $G(\alpha, \beta) \in \mathscr{V}_{0}(X)$ is continuous at $y$, we can find an open neighborhood $U \ni y$ and a trivialization $\phi_{U}:\left.\mathscr{V}\right|_{U} \rightarrow U \times V$, such that

$$
\left\|\mathrm{pr}_{2} \circ \phi_{U}(G(\alpha, \beta)(y))-\mathrm{pr}_{2} \circ \phi_{U}\left(G(\alpha, \beta)\left(y^{\prime}\right)\right)\right\| \leq \varepsilon
$$

for all $y^{\prime} \in U$. In view of our above observation this proves continuity of $y \mapsto$ $\varphi_{y}(G)$ with respect to the quasi-strict topology, since applying Lemma 2.18 one has

$$
\begin{aligned}
\| a \triangleleft & \left(\operatorname{pr}_{2} \circ \psi_{U}\left(\varphi_{y}(G)\right)-\operatorname{pr}_{2} \circ \psi_{U}\left(\varphi_{y^{\prime}}(G)\right)\right) \triangleright b \| \\
& =\left\|\operatorname{pr}_{2} \circ \psi_{U}\left(\varphi_{y}(G)\right)(a, b)-\operatorname{pr}_{2} \circ \psi_{U}\left(\varphi_{y^{\prime}}(G)\right)(a, b)\right\| \\
& =\left\|\operatorname{pr}_{2} \circ \phi_{U}\left(\varphi_{y}(G)(a, b)\right)-\operatorname{pr}_{2} \circ \phi_{U}\left(\varphi_{y^{\prime}}(G)(a, b)\right)\right\| \\
& =\left\|\operatorname{pr}_{2} \circ \phi_{U}(G(\alpha, \beta)(y))-\operatorname{pr}_{2} \circ \phi_{U}\left(G(\alpha, \beta)\left(y^{\prime}\right)\right)\right\| \leq \varepsilon .
\end{aligned}
$$

By the independence of the bound in (14) the section constructed above is also bounded. Therefore

$$
S: Q M\left(\mathscr{V}_{0}(X)\right) \longrightarrow C_{b}(X, Q M(\mathscr{V})), \quad G \mapsto\left(y \mapsto \varphi_{y}(G)\right) .
$$

is well-defined, linear and satisfies $\|S(G)\| \leq\|G\|$. For the inverse direction consider

$$
\begin{aligned}
& \Phi: C_{b}(X, Q M(\mathscr{V})) \rightarrow Q M\left(\mathscr{V}_{0}(X)\right), \\
& \Phi(F)(\alpha, \beta)(x):=F(x)(\alpha(x), \beta(x)) .
\end{aligned}
$$

First, we have to check that the element $\Phi(F)(\alpha, \beta)$ belongs to $\mathscr{V}_{0}(X)$, i.e. that the function

$$
x \mapsto F(x)(\alpha(x), \beta(x))
$$

vanishes at infinity and is continuous. For any $\varepsilon>0$ there is a compact $K \subset X$ such that $\|\alpha(x)\|<\varepsilon$ and $\|\beta(x)\|<\varepsilon$ for $x \in X \backslash K$. Then

$$
\begin{aligned}
\|\Phi(F)(\alpha, \beta)(x)\| & =\|F(x)(\alpha(x), \beta(x))\| \\
& \leq\|F(x)\|\|\alpha(x)\|\|\beta(x)\| \\
& \leq\|F\| \varepsilon^{2}
\end{aligned}
$$

for $x \in X \backslash K$ proving that it vanishes at infinity.

For the verification of continuity let $\varepsilon>0$ and $x \in X$. There is a neighborhood $U_{1}$ of $x$ such that

$$
\|\alpha(x)-\alpha(y)\|<\varepsilon \text { and }\|\beta(x)-\beta(y)\|<\varepsilon \text { whenever } y \in U_{1} .
$$

On the other hand by Lemma 2.18 there is a neighborhood $U_{2} \subset U_{1}$ of $x$ such that

$$
\begin{aligned}
& \left\|\left(\left.\mathrm{pr}_{2} \circ \psi_{U_{2}} \circ F\right|_{U_{2}}\right)(x)(\alpha(x), \beta(x))-\left(\left.\mathrm{pr}_{2} \circ \psi_{U_{2}} \circ F\right|_{U_{2}}\right)(y)(\alpha(x), \beta(x))\right\| \\
& \quad=\left\|\mathrm{pr}_{2} \circ \phi_{U_{2}}(F(x)(\alpha(x), \beta(x)))-\mathrm{pr}_{2} \circ \phi_{U_{2}}(F(y)(\alpha(x), \beta(x)))\right\|<\varepsilon
\end{aligned}
$$


whenever $y \in U_{2}$. One has

$$
\begin{aligned}
\left\|\mathrm{pr}_{2} \circ \phi_{U_{2}}(\Phi(F)(\alpha, \beta)(x))-\mathrm{pr}_{2} \circ \phi_{U_{2}}(\Phi(F)(\alpha, \beta)(y))\right\| \\
=\left\|\mathrm{pr}_{2} \circ \phi_{U_{2}}(F(x)(\alpha(x), \beta(x)))-\mathrm{pr}_{2} \circ \phi_{U_{2}}(F(y)(\alpha(y), \beta(y)))\right\| \\
\leq\left\|\mathrm{pr}_{2} \circ \phi_{U_{2}}(F(x)(\alpha(x), \beta(x)))-\mathrm{pr}_{2} \circ \phi_{U_{2}}(F(y)(\alpha(x), \beta(x)))\right\| \\
\quad+\left\|\mathrm{pr}_{2} \circ \phi_{U_{2}}(F(y)(\alpha(x), \beta(x)))-\mathrm{pr}_{2} \circ \phi_{U_{2}}(F(y)(\alpha(y), \beta(x)))\right\| \\
\quad+\left\|\mathrm{pr}_{2} \circ \phi_{U_{2}}(F(y)(\alpha(y), \beta(x)))-\mathrm{pr}_{2} \circ \phi_{U_{2}}(F(y)(\alpha(y), \beta(y)))\right\| \\
\leq \varepsilon+\|F\|\|\beta\| \varepsilon+\|F\|\|\alpha\| \varepsilon
\end{aligned}
$$

for $y \in U_{2}$, which proves continuity of $\Phi(F)(\alpha, \beta)$. Together with the norm estimates (15), this completes the proof of well-definedness of $\Phi$. Clearly, $\Phi$ is the inverse of $S$. Moreover, the inequalities (14) and (15) ensure that $S$ is an isometry.

REMARK 5.5. The evaluation map $\varphi_{y}$ used in the proof coincides with the extension of

$$
\varphi_{y}: \mathscr{V}_{0}(X) \longrightarrow \mathscr{V}_{y}
$$

with respect to the quasi-strict topology.

Let $\mathscr{V}$ be a bundle of right Hilbert $B$-modules for a $C^{*}$-algebra $B$. By a similar construction as the one given above there is a bundle $L M(\mathscr{V})$ of left multipliers and a bundle $M(\mathscr{V})$ of double multipliers. The above arguments may be used to prove the following analogue of Theorem 5.4 for left and (double) multipliers.

THEOREM 5.6. There are the following isometric B-module isomorphisms

$$
\begin{aligned}
L M\left(\mathscr{V}_{0}(X)\right) & \cong C_{b}(X, L M(\mathscr{V})), \\
M\left(\mathscr{V}_{0}(X)\right) & \cong C_{b}(X, M(\mathscr{V})),
\end{aligned}
$$

where $\operatorname{LM}(\mathscr{V})$ (resp., $M(\mathscr{V}))$ on the right-hand side are equipped with the left strict (resp., strict) topology.

ACKNOWLEDGEMENT. The presented work was partially supported by the joint RFBR and Deutsche Forschungsgemeinschaft (DFG) project (RFBR grant 07-01-91555 / DFG project "K-Theory, $C^{*}$-algebras, and Index theory"). AP was partially supported by the RFBR (grant 08-01-00867). The research was carried out during the visit of the first author to the Georg-AugustUniversität Göttingen, and he appreciates its hospitality a lot.

The authors are grateful to the referee for his/her valuable suggestions. 


\section{REFERENCES}

1. Akemann, C. A., Pedersen, G. K., and Tomiyama, J., Multipliers of $C^{*}$-algebras, J. Functional Analysis 13 (1973), 277-301.

2. Bakić, D., Guljaš, B., Extensions of Hilbert $C^{*}$-modules, Houston J. Math. 30 (2004), 537558.

3. Brown, L. G., Mingo, J. A., Shen, N.-T., Quasi-multipliers and embeddings of Hilbert $C^{*}$ bimodules, Canad. J. Math. 46 (1994), 1150-1174.

4. Busby, R. C., Double centralizers and extensions of $C^{*}$-algebras, Trans. Amer. Math. Soc. 132 (1968), 79-99.

5. Frank, M., A set of maps from $K$ to $\operatorname{End}_{A}\left(l_{2}(A)\right)$ isomorphic to $\operatorname{End}_{A(K)}\left(l_{2}(A(K))\right)$. Applications, Annals Global Anal. Geom. 3 (1985), 155-171.

6. Frank, M., Kirchberg, E., On conditional expectations of finite index, J. Operator Theory 40 (1998), 87-111.

7. Frank, M., Manuilov, V. M., and Troitsky, E. V., On conditional expectations arising from group actions, Z. Anal. Anwendungen 16 (1997), 831-850.

8. Frank, M., Pavlov, A. A., Strict essential extensions of $C^{*}$-algebras and Hilbert $C^{*}$-modules, J. Operator Theory 64 (2010), 103-116.

9. Kasparov, G. G., Hilbert $C^{*}$-modules: Theorems of Stinespring and Voiculescu, J. Operator Theory 4 (1980), 133-150.

10. Kasparov, G. G., The operator $K$-functor and extensions of $C^{*}$-algebras, Math. USSR Izv. 16 (1981), 513-572.

11. Lance, E. C., Hilbert $C^{*}$-modules. A Toolkit for Operator Algebraists, London Math. Soc. Lecture Note 210, Cambridge Univ. Press, Cambridge 1995.

12. Manuilov, V. M., Troitsky, E. V., Hilbert $C^{*}$-modules, Transl. Math. Monogr. 226, Amer. Math. Soc., Providence, RI 2005.

13. Pavlov, A. A., Algebras of multiplicators and spaces of quasimultiplicators, Moscow Univ. Math. Bull. 53:6 (1998), 13-16.

14. Pavlov, A. A., Description of the set of homomorphisms from the Hilbert module $C_{0}(X, \mathcal{M})$ to its dual module $C_{0}(X, M)^{\prime}$, Moscow Univ. Math. Bull. 57:3 (2002), 43-46.

15. Pedersen, G. K., $C^{*}$-algebras and Their Automorphism Groups, London Math. Soc. Monogr. 14, Academic Press, London 1979.

16. Raeburn, I., Thompson, S. J., Countably generated Hilbert modules, the Kasparov stabilisation theorem, and frames with Hilbert modules, Proc. Amer. Math. Soc. 131 (2002), $1557-1564$.

17. Raeburn, I., Williams, D. P., Morita Equivalence and Continuous-Trace $C^{*}$-Algebras, Math. Surveys Monogr. 60, Amer. Math. Soc., Providence, RI 1998.

18. Watatani, Y., Index for $C^{*}$-subalgebras, Mem. Amer. Math. Soc. 83 (1990), no. 424.

ALL-RUSSIAN INSTITUTE OF SCIENTIFIC

AND TECHNICAL INFORMATION

RUSSIAN ACADEMY OF SCIENCES (VINITI RAS)

USIEVICHA STR. 20

125190 MOSCOW A-190

RUSSIA

E-mail: axpavlov@gmail.com
MATHEMATISCHES INSTITUT

GEORG-AUGUST-UNIVERSITÄT

BUNSENSTRAßE 3-5

D-37073 GÖTTINGEN

GERMANY

E-mail: pennig@math.uni-goettingen.de schick@uni-math.gwdg.de 